\title{
Multi-axial response of idealized cermets
}

\author{
E. G. Pickering, E. Bele \& V. S. Deshpande \\ Department of Engineering, University of Cambridge, Trumpington Street, Cambridge, United \\ Kingdom
}

\begin{abstract}
The yield response of two idealized cermets comprising mono and bi-disperse steel spheres in a $\mathrm{Sn} / \mathrm{Pb}$ solder matrix has been investigated for a range of axisymmetric stress states. Proportional stress path experiments are reported, from which are extracted the initial yield surfaces and their evolution with increasing plastic strain. The initial yield strength is nearly independent of the hydrostatic pressure but the strain hardening rate increases with stress triaxiality up to a critical value. For higher triaxialities, the responses are independent of hydrostatic pressure. Multi-axial measurements along with X-ray tomography were used to demonstrate that the deformation of these idealized cermets occurs by two competing mechanisms: (i) a granular flow mechanism that operates at low levels of triaxiality, where volumetric dilation occurs under compressive stress states, and (ii) a plastically incompressible mechanism that operates at high stress triaxialities. A phenomenological viscoplastic constitutive model that incorporates both the deformation mechanisms is presented. While such multi-axial measurements are difficult for commercial cermets with yield strengths on the order of a few GPa, the form of their constitutive relation is expected to be similar to that of the idealized cermets presented here.
\end{abstract}

Keywords: cermets; yield surface; constitutive law; plasticity theory 


\section{Introduction}

Cermets are composite materials that contain a high volume fraction of ceramic particulates (typically carbides, nitrides, and oxides, in the range of $50 \%-95 \%$ by volume) within a ductile metal binder phase (e.g. Mo, Ni, Co, Al) $[1,2]$. They offer a good compromise between the hardness of ceramics and toughness of metals, e.g. typical values for WC/Co composites are in the range of $500-2000 \mathrm{HV}$ and $8-20 \mathrm{MPa} \mathrm{m}^{1 / 2}$ respectively [3]. This combination of mechanical properties makes them suitable for lightweight ballistic armour applications, where the high hardness value results in the erosion/destruction of the projectile [4], and the improved toughness increases the ability of the structure to sustain multiple impacts [5]. More commonly, cermets are used as hard coatings for tools and high-pressure components, in order to reduce wear and increase the service life $[6,7]$.

The elastic properties of ceramic particle reinforced metals with low particulate volume fractions (e.g. less than 50\%) have received extensive attention. These include their uniaxial response [8-10] as well as multi-axial behaviour [11]. By contrast, investigations of the inelastic properties of cermets have mainly been restricted to the influence of the particulate volume fraction on the uniaxial compressive response $[10,12]$, indentation hardness, and toughness. Little information exists about the dependence of the multi-axial response of these materials on stress triaxiality, possibly because the extremely high yield strength impedes instrumented measurements of the deformations involved. Several studies have used metal jacket confinement tests $[13,14]$ to provide a qualitative estimate of the behaviour of these materials under multi-axial stress states. These studies suggest that both the compressive strength and ductility increase with increasing lateral confinement $[13,14]$. Notched tensile specimens have also been used to examine the influence of triaxial stress on the ductility and failure of cermets, with an increase in tensile triaxiality observed to increase the failure stress 
and decrease the failure strain [15]. However, since the precise multi-axial stress state is not straightforward to extract, such measurements cannot be used to estimate the yield surfaces of cermets or the corresponding plastic flow rules.

Uniaxial compression tests of cermets suggest that they behave like high cohesive strength granular materials with shear band formation and associated dilatancy $[16,17]$. For example, Getting et al. [12] observed that WC/Co cermets dilate under uniaxial loading, and Bele and Deshpande [16] demonstrated via experiments on idealized cermets that this dilation was primarily confined to shear bands. These deformation mechanisms are similar to those of granular media [17], where an initial compaction phase occurs as inter-particulate voids collapse and the particulate density increases. The newly-packed granular assembly contains a network of force chains formed by contacting particles [17]. The maximum compressive strength of the material is governed by the buckling of these force chains and the formation of a localised shear band [16], and volumetric dilation within the shear band is due to the progressive buckling of the force chains. Correspondingly, increasing the confining pressure will delay the onset of volumetric dilation within the shear band [13,14,17]. The effect of these force chains is further illustrated by the marked difference in compressive and tensile failure stresses observed for high volume fraction cermets [18]. By contrast low volume fraction cermets of the same composition displayed negligible tension-compression asymmetry.

In order to investigate the mechanical properties of cermets in more detail, idealized model materials have been used, e.g. composites comprising steel spheres in a soft $\mathrm{Sn} / \mathrm{Pb}$ solder matrix [16]. These idealized materials possess the same particle/matrix contrast in elastic moduli and yield strengths as commercial cermets, but have a simpler microstructure and 
significantly lower macroscopic yield strength, thus facilitating standard instrumented laboratory-based mechanical testing. Uniaxial compression of these materials has demonstrated that (a) percolation of the steel spheres and the associated formation of force chains leads to a compressive strength that is significantly higher than what is predicted by models of uniformly distributed aggregates, and (b) deformation under uniaxial compression is due to the formation of shear bands, with significant dilation occurring within these bands [19].

There is thus considerable evidence that commercial cermets behave like granular materials $[12,16,17,19]$. Therefore, their multi-axial yield response is not expected to be governed solely by the von-Mises equivalent stress. In this study, we take advantage of the relatively low compressive strength of idealized cermets to measure their multi-axial plastic response, with the aim of elucidating the constitutive behaviour of commercial materials. A triaxial load cell is used to conduct axisymmetric compressive experiments over a range of stress states (triaxialities). The experimental set-up allows simultaneous control of the stress triaxiality, and direct measurement of axial and radial strains. These measurements, along with X-ray tomography, are used to clarify the dependence of deformation mechanisms on the triaxial stress state, and to extract a phenomenological constitutive model for the yield surface and plastic flow rule of these materials.

\section{Experimental investigation}

The overall aims of the experimental program are to (i) determine the stress-strain responses of the idealized cermets under proportional compressive axisymmetric loading, and (ii) investigate the shape of the initial yield surface and its evolution under proportional compressive load paths. 


\subsection{Materials and manufacture}

Two types of idealized cermets, comprising AISI 52100 steel spheres in a $\mathrm{Sn} / \mathrm{Pb}$ solder matrix ( $\mathrm{Sn}$ 60, Pb 38, Ag 2 wt.\%), were manufactured as in [15]: (i) mono-dispersed specimens, with spheres of $2 \mathrm{~mm}$ diameter packed to a volume fraction $V_{f} \approx 65 \%$ in the solder matrix, and (ii) bi-dispersed specimens, with spheres of diameters $4 \mathrm{~mm}$ and $0.5 \mathrm{~mm}(70 \%$ and $30 \%$ by volume respectively ${ }^{\mathrm{a}}$ ), packed to an overall volume fraction $V_{f} \approx 0.75$.

Cylindrical specimens of diameter $18.5 \mathrm{~mm}$ and height $40 \mathrm{~mm}$ were manufactured for both types of idealized cermets. The spheres were first packed into a cylindrical crucible of the appropriate diameter and vibrated under a low applied axial compressive stress of $\approx 0.1 \mathrm{MPa}$ to maximize the packing density. High temperature magnets were then placed around the periphery of the crucible to maintain the skeleton structure of the steel spheres, and solder powder (of average particle size $25-38 \mu \mathrm{m}$ ) was infiltrated into the interstitial sites. A small amount of $\mathrm{ZnCl}$ flux was added to improve interfacial adhesion, and the assembly was pressure-cast at a pressure of $\approx 0.1 \mathrm{MPa}$ and temperature of $200^{\circ} \mathrm{C}$ for $1 \mathrm{hr}$.

Optical images of the cross-section of representative mono and bi-disperse specimens are included in Fig. 1. Some voids are visible in both types of specimens; X-ray tomography measurements (discussed in Section 3) indicated that the void volume fraction was in the range of $1.5 \%-5 \%$ in both types of specimens. Vickers hardness tests were conducted to measure the mechanical properties of the two phases and revealed that the AISI 52100 steel had a Young's modulus of $210 \mathrm{GPa}$ and yield strength of $2.1 \mathrm{GPa}$, while the $\mathrm{Sn} / \mathrm{Pb}$ solder had corresponding modulus and yield strength values of $32 \mathrm{GPa}$ and $39 \mathrm{MPa}$ respectively

\footnotetext{
${ }^{a}$ Readers are referred to [20] for a detailed discussion on the random packing of spheres, including maps that specify the sphere size distributions and volume fractions required in order to achieve a specified overall volume fraction of spheres.
} 
(uniaxial compression tests on the matrix material reported in [16] confirmed these properties of the $\mathrm{Sn} / \mathrm{Pb}$ solder).

\subsection{Apparatus and measurement protocol}

A high-pressure apparatus (Fig. 2a) was used to subject the specimens to triaxial compression tests. It consists of a pressure cell with a maximum capacity of $100 \mathrm{MPa}$, and a piston for the application of axial force. Hydraulic fluid was used as the pressurising medium, and axial load was applied by displacing the piston via a screw-driven test machine. A submerged load cell provided readings of the axial load independently of the pressure of the surrounding fluid. Two linear variable differential transformer (LVDT) transducers were attached to the specimen as shown in Fig. 2a in order to measure the axial displacement imposed on the specimen. A third LVDT was attached to the mid-height of the specimen to measure the change in specimen diameter.

The applied stresses are sketched in Fig. 2b: $p$ denotes the fluid pressure and $\sigma_{a}$ the axial stress, defined as the ratio of the applied axial load (as measured by the submerged load cell) to the initial cross-sectional area of the specimen. With $p$ taken to be positive in compression, the mean (hydrostatic) stress $\sigma_{m}$, and von Mises effective stress $\sigma_{e}$ are

$$
\sigma_{m}=-p+\frac{\sigma_{a}}{3}
$$

and $\sigma_{e}=\left|\sigma_{a}\right|$, respectively. Note that the radial Cauchy stress on the specimen equals the fluid pressure $p$. The corresponding work-conjugate strains (mean strain $\varepsilon_{m}$ and effective strain $\varepsilon_{e}$ ) are given in terms of the axial strain $\varepsilon_{a}$ and radial strain $\varepsilon_{r}$ as

$$
\varepsilon_{m}=2 \varepsilon_{r}+\varepsilon_{a}
$$

and 


$$
\varepsilon_{e}=\frac{2}{3}\left|\varepsilon_{r}-\varepsilon_{a}\right|
$$

respectively.

Prior to the start of each test, the specimen was consolidated within the triaxial cell by applying a pure hydrostatic pressure $p=100 \mathrm{MPa}$ : this consolidation step was found to improve the repeatability of the measurements. The majority of tests were conducted along proportional stress paths with the direction of the stress path defined by the relation $\eta=$ $\sigma_{m} / \sigma_{e}$. The triaxiality parameter $\eta$ can take values over the range $\eta=1 / 3$ (uniaxial compression) to $\eta=\infty$ (hydrostatic compression). In the proportional loading experiments, the fluid pressure $p$ and the axial stress $\sigma_{a}$ were controlled in unison to increase $\sigma_{e}$ and $\sigma_{m}$ while keeping $\eta$ constant. All experiments reported here were conducted for an applied loading rate $\sigma_{e}=10 \mathrm{kPa} \mathrm{s}^{-1}$.

For these proportional stress path tests it was unnecessary to seal the specimens from the surrounding fluid, as the specimen porosity was sufficiently low and discontinuous to prevent fluid penetration. However, some additional non-proportional tests were also conducted along paths of low, followed by high triaxiality. In these tests, dilation during the low triaxiality phase created an additional connected porosity and thus in those tests it was necessary to use a rubber membrane to seal the specimens, following the protocol outlined in [21]. Since the rubber membrane introduced large uncertainties in the measurement of radial displacements, only the axial displacements were recorded for these tests. 


\section{Triaxial response of idealized cermets}

We report measurements of the responses of mono and bi-dispersed idealized cermets. In all cases, at-least three repeat measurements were conducted for each test; the level of scatter was within $10 \%$.

\subsection{Mono-disperse cermets}

The measured equivalent stress-strain $\left(\sigma_{e}-\varepsilon_{e}\right)$ compressive responses for triaxiality values in the range $\eta=1 / 3$ (uniaxial compression) to $\eta=1.5$ are plotted in Fig. 3a, and the corresponding variation of the mean strain $\varepsilon_{m}$ with the effective strain $\varepsilon_{e}$ are included in Fig. 3b. The pressure limit of the triaxial cell $(p=100 \mathrm{MPa})$ imposes a maximum achievable strain under proportional loading: at a triaxiality $\eta=1.5$ this maximum strain was $\varepsilon_{e} \approx$ 0.035 for the mono-disperse specimens, and at higher triaxialities the inelastic response was not accessible.

The stress-strain curves of Fig. 3a show that both the initial elastic response and the yield stress (defined at the $0.2 \%$ strain offset) are largely independent of $\eta$. However, the subsequent plastic hardening response is strongly affected by the triaxiality: while the uniaxial response is approximately perfectly plastic, the plastic hardening rate increases up to $\eta=1$. Intriguingly, the $\sigma_{e}-\varepsilon_{e}$ curves become virtually identical for $\eta \geq 1$. From these tests, it is clear that the mechanical response of cermets is strongly affected by the stress triaxiality $\eta$, and is fundamentally different from the behaviour of metals.

Some insight into the causes of this difference is given by the corresponding $\varepsilon_{m}-\varepsilon_{e}$ curves included in Fig. 3b. In the low triaxiality limit $(1 / 3 \leq \eta \leq 0.75)$, two distinct regimes are seen: (i) an initial compaction regime where $d \varepsilon_{m} / d \varepsilon_{e}<0$, and (ii) a dilation regime with 
$d \varepsilon_{m} / d \varepsilon_{e}>0$, wherein the volume of the specimen increases. While dilation commences very early under uniaxial compression (at approximately the yield strain of $\varepsilon_{e}=0.5 \%$ ), the dilatory regime is delayed at higher triaxialities, e.g. starting at $\varepsilon_{e}=3.5 \%$ in the tests with $\eta=0.75$. We argue that similar to granular media (see for example Schofield [22]), the work done against the hydrostatic pressure by the dilatory response of the cermet results in the plastic hardening rate increasing with $\eta$, as seen in Fig. 3a. With further increases in triaxiality, the dilatory part of the response is further delayed; over the strain levels tested here no dilation occurred in the regime $\eta \geq 1$, and the $\varepsilon_{m}-\varepsilon_{e}$ curves were nearly identical (Fig. 3b). Since the plastic response is now nearly volumetrically incompressible, the stressstrain curves are uniquely described by the von-Mises effective stress with no sensitivity to $\eta$ as seen seen in Fig. 3a. It is worth emphasizing here that the compaction-dilation behaviour seen here for idealized cermets at low triaxialities has been observed under uniaxial compression in commercial cermets with particulate volume fractions between $80-92 \%$; see for example Getting et al. [12]. Similarly, the decrease in dilatancy with increasing triaxiality is also observed in granular materials [17].

In order to gain further insight into the influence of stress triaxiality on deformation mechanisms, we include in Figs. 4a and 4b X-ray tomographic images across the longitudinal mid-section of specimens tested with $\eta=1 / 3$ and 1.0, respectively. For each case, the sections show the undeformed specimen (at $\varepsilon_{e}=0$ ) and after compression to $\varepsilon_{e}=0.05$ (with elastic recovery typically less than $0.1 \%$ ). First consider the $\eta=1 / 3$ case. Deformation occurs by a combination of shear and overall volumetric expansion. The volumetric expansion is indicated in the highlighted region in Fig. 4a showing the opening of new voids. However, we emphasize that some void collapse is also observed in the $\eta=1 / 3$ case consistent with the initial compaction regime shown in Fig. 3b. Now consider the high 
triaxiality case of $\eta=1$ shown in Fig. 4b. No localised deformation or shear band is observed, and the deformation is now homogeneously distributed through the specimen. A magnified view of a region within the specimen is also included in Fig. 4b. Collapsing voids marked in this region are consistent with the measured compaction of the specimen.

In a qualitative sense, this change in the deformation mechanism is rationalised as follows. The plastic deformation of the cermet can occur by two possible deformation mechanisms: (i) an overall dilatory mechanism with the formation of regions of high void concentrations and (ii) an overall incompressible mechanism with a corresponding homogenous deformation. The operative mechanism is that which occurs at a lower stress level. At low triaxialities the dilatory mechanism occurs at a lower collapse stress. However, with increasing triaxiality the work needed to dilate the specimen against the imposed hydrostatic pressure also increases, making the incompressible mechanism more favourable. This qualitative understanding is quantified in Section 4, when the collapse locus of each of these mechanisms is inferred from the multi-axial measurements.

\subsubsection{Non-proportional stress paths}

A limited number of tests were conducted in order to quantify the effect of non-proportional stress paths on the measured response of the mono-disperse cermet. Here we report two types of such tests, illustrated by the insets in Figs. 5a and $5 \mathrm{~b}$ respectively:

(i) The $\eta(1 / 3 \rightarrow 1)$ test. The specimen is loaded in uniaxial compression and unloaded to a residual axial strain $\varepsilon_{a}=0.035$. The specimen is then reloaded under a $\eta=1$ stress path up to a total strain $\varepsilon_{a}=0.070$. 
(ii) The $\eta(1 \rightarrow 1 / 3)$ test. The specimen is loaded along a $\eta=1$ stress path and unloaded to a residual axial strain $\varepsilon_{a}=0.010$. The specimen is then reloaded in uniaxial compression up to a total strain $\varepsilon_{a}=0.035$

The measurements of the $\sigma_{e}-\varepsilon_{a}$ histories for the $\eta(1 / 3 \rightarrow 1)$ and $\eta(1 \rightarrow 1 / 3)$ tests are shown in Figs. 5a and 5b, respectively, along with the corresponding proportional loading measurements with $\eta=1 / 3$ and 1 from Fig. 3a. First consider Fig. 5a. After unloading from the uniaxial compression path and reloading along $\eta=1$, the $\sigma_{e}-\varepsilon_{a}$ curve is seen to re-join the fully proportional $\eta=1$ stress history included in Fig. 5a from Fig. 3a. This indicates negligible non-proportional loading effects of the measured idealized cermet response. The same conclusion is also drawn for the other non-proportional stress path considered in Fig. 5b. We thus conclude that the yield strength of the cermet is well characterised by the stress triaxiality $\eta$ and the accumulated strain $\varepsilon_{e}$ without any significant load path history effects.

\subsubsection{Yield surfaces}

The initial yield surface of these specimens and its evolution with plastic strain is summarized in Fig. 6a. The initial yield strength is obtained by the $0.2 \%$ strain offset in the $\sigma_{e}-\varepsilon_{e}$ curves. A plot of the stress pair $\left(\sigma_{m}, \sigma_{e}\right)$ corresponding to this definition of the yield point at all the values of $\eta$ tested here is shown in Fig. 6a, and marks the locus of the initial yield stresses. In addition, we include in Fig. 6a loci of $\left(\sigma_{m}, \sigma_{e}\right)$ at three selected values of applied effective plastic strain $\varepsilon_{e}^{p}$. To help guide the eye, curves using a functional form detailed in Section 4 are included in Fig. 6a. Recall that non-proportional stress path effects are negligible and the yield strength of the cermet is well characterized by the stress triaxiality $\eta$ and the accumulated strain $\varepsilon_{e}^{p}$. Thus, it is reasonable to assume that the loci plotted in Fig. 6a approximate the yield surfaces of the cermet under axisymmetric loading. 
The evolution of the yield surfaces seen in Fig. 6a follows the $\sigma_{e}-\varepsilon_{e}$ responses discussed in Fig. 3a. At low applied strains $\varepsilon_{e}$, the yield strength is approximately independent of $\eta$ and thus the yield surface is nearly a horizontal line in $\sigma_{e}-\sigma_{m}$ space. With increasing applied strain $\varepsilon_{e}$, there is significant plastic hardening, especially in the high triaxiality cases. Therefore, two distinct regimes in the yield surfaces emerge:

(i) At low triaxiality the yield surface has a Drucker-Prager [23] characteristic with the yield strength being pressure dependent. This is consistent with observations for granular media.

(ii) At high triaxialities the yield surface again asymptotes to a von-Mises surface with the yield strength independent of pressure.

The direction of the plastic strain rate vector $\left(\dot{\varepsilon}_{e}, \dot{\varepsilon}_{m}\right)$ at selected loading points are included in Fig. 6a, with the $\dot{\varepsilon}_{e}$ and $\dot{\varepsilon}_{m}$ axes co-incident with the $\sigma_{e}$ and $\sigma_{m}$ axes respectively. To within the measurement error in defining $\left(\dot{\varepsilon}_{e}, \dot{\varepsilon}_{m}\right)$ and the data scatter, we conclude that plastic straining is normal to the yield surfaces.

\subsection{Bi-disperse cermets}

The responses of bi-disperse cermets as characterized by the $\sigma_{e}-\varepsilon_{e}$ and $\varepsilon_{m}-\varepsilon_{e}$ curves are plotted in Figs. 7a and $7 \mathrm{~b}$ respectively for selected values of $\eta$. The strength of the bi-disperse cermet for any given $\eta$ is higher than the corresponding mono-disperse case; compare Figs. 7a and 3a. This is primarily a particle volume fraction effect $\left(V_{f}=0.75 \mathrm{vs.} V_{f}=0.65\right.$, for bi- and mono-disperse materials respectively), and has been reported extensively in the context of commercial cermets $[10,24]$. Bele and Deshpande [16] found similar effects in idealized cermets. They argued that as $V_{f}$ increased beyond the maximum random packing density of $65 \%$ of mono-sized spheres, increased percolation of the inclusion phase resulted 
in the formation of force chains which were the primary cause for the marked strengthening effect.

While the strength of the bi-disperse cermets is higher than the mono-disperse cermets, a comparison of Figs. 3 and 7 suggests that the qualitative nature of the response remains unchanged. Thus, similar to the mono-disperse cermets, the plastic hardening rate initially increases with $\eta$ and then remains unchanged for $\eta \geq 1$ (Fig. 7a). Similarly, the $\varepsilon_{m}-\varepsilon_{e}$ curves show a compaction/dilation sequence at low triaxialities, and only compaction followed by an incompressible response at higher triaxialities (Fig. 7b). The corresponding yield surfaces (initial yield, and at $\varepsilon_{e}^{p}=0.01$ ) are included in Fig. $6 \mathrm{~b}$ along with the direction of the plastic strain rate vectors at each loading point. Again, the qualitative nature of the yield surfaces and plastic flow directions are similar to that of the mono-disperse materials (also included in Fig. $6 \mathrm{~b}$ for easy comparison), except that the yield strengths are higher.

\section{A phenomenological constitutive model}

Based on the experimental measurements reported above, we propose a phenomenological constitutive model for idealized cermets. Two deformation modes are observed in the experiments, corresponding to a granular-type dilatory mechanism that is pressure-dependent, and an incompressible mechanism that is only a function of the von-Mises effective stress. We shall subsequently refer to these mechanisms as the granular and incompressible mechanisms, respectively. The yield surface of the idealized cermet can then be viewed as the inner envelope of the set of lines in $\sigma_{m}-\sigma_{e}$ space corresponding to critical stressing of the two separate deformation mechanisms as sketched in Fig. 8a. The intersection of the two lines results in a corner in the yield surface. In order to alleviate the numerical difficulties associated with corners, and also taking into account that the latter are not present in the 
experimental data (Fig. 6), we proceed to present a viscoplastic constitutive model that naturally rounds off the corners [25]. Following Peirce et al. [26] this viscoplastic model can be made nearly rate-insensitive by an appropriate choice of parameters.

An isotropic constitutive model is developed, wherein we assume that plastic flow only depends on the first two stress invariants $\sigma_{m}$ and $\sigma_{e}$ and is independent of the third invariant $J_{3}=\left(\sigma_{i j}^{\prime} \sigma_{j k}^{\prime} \sigma_{k i}^{\prime}\right)^{1 / 3}$, where $\sigma_{i j}^{\prime}$ is the deviatoric stress tensor. The flow potential $\Phi$ is written as the sum of two potentials $\Phi_{G}$ and $\Phi_{I}$ corresponding to the granular and incompressible mechanisms, respectively. Thus, we write $^{\mathrm{b}} \Phi \equiv \Phi_{I}+\Phi_{G}$, where $\Phi_{I}$ is the von-Mises viscoplastic potential given as

$$
\Phi_{I} \equiv \frac{\sigma_{0} \dot{\varepsilon}_{0}}{n+1}\left(\frac{\sigma_{e}}{\sigma_{0}}\right)^{n+1}
$$

In Eq. 4.1, $\sigma_{0}\left(\varepsilon_{e}^{p}\right)$ represents the strain hardening of the incompressible mechanism in terms of the effective plastic strain $\varepsilon_{e}^{p}, \dot{\varepsilon}_{0}$ is a reference strain rate such that $\dot{\varepsilon}_{e}^{p}=\dot{\varepsilon}_{0}$ at $\sigma_{e}=\sigma_{0}$, and $n$ is the strain rate sensitivity exponent. The granular potential $\Phi_{G}$ is written in terms of an equivalent stress

$$
\hat{\sigma} \equiv \begin{cases}\frac{\sigma_{e}+\mu \sigma_{m}}{1-\frac{\mu}{3}} & \sigma_{e} \geq-\mu \sigma_{m} \\ 0 & \text { otherwise }\end{cases}
$$

where $\mu$ is the so-called friction coefficient of granular media, equal to the slope of the yield line in $\sigma_{m}-\sigma_{e}$ space as shown in Fig. 8a. We then write $\Phi_{G}$ as

$$
\Phi_{G} \equiv \frac{\sigma_{Y} \dot{\varepsilon}_{0}}{n+1}\left(\frac{\hat{\sigma}}{\sigma_{Y}}\right)^{n+1}
$$

\footnotetext{
${ }^{\mathrm{b}}$ The curves fitted to the data in Fig. 6 are contours of the form $\Phi=C$ with $n=20$ and the remaining constants, including $C$, chosen to give a good fit to the data.
} 
with $\sigma_{Y}$ as the uniaxial yield strength of the idealized cermet. Recall that under uniaxial compression the cermets displayed negligible strain hardening and therefore we assume that $\sigma_{Y}$ is independent of the plastic strain $\varepsilon_{e}^{p}$. Following the data in Fig. 6 we assume plastic normality with the plastic strain rate given by

$$
\dot{\varepsilon}_{i j}^{p}=\frac{\partial \Phi}{\partial \sigma_{i j}} .
$$

The constitutive description is then completed by specifying the total strain rate $\dot{\varepsilon}_{i j}$ as the sum of the elastic and plastic strain rates such that

$$
\dot{\varepsilon}_{i j}=\dot{\varepsilon}_{i j}^{e}+\dot{\varepsilon}_{i j}^{p}
$$

and the elastic strain rate follows from the isotropic Hooke's law

$$
\dot{\varepsilon}_{i j}^{e}=\frac{1+v}{E} \dot{\sigma}_{i j}-\frac{v}{E} \dot{\sigma}_{k k} \delta_{i j}
$$

where $E$ and $v$ are the Young's modulus and Poisson's ratio, respectively while $\delta_{i j}$ denotes the Kronecker delta.

\subsection{Calibration of model parameters}

The key material parameters are directly obtained from the following tests:

(i) The elastic constants $E$ and $v$ are obtained in a standard manner, e.g. from a uniaxial compression test, by measuring the unloading modulus and lateral expansion of the specimen prior to first yield.

(ii) The uniaxial compression strength $\sigma_{Y}$ also follows directly from a uniaxial compression experiment, with $\sigma_{Y}$ taken as the measured plateau in the uniaxial compression stress versus strain curve; see for example Fig. 3a. 
(iii) The strain hardening response $\sigma_{0}\left(\varepsilon_{e}^{p}\right)$ is the measured $\sigma_{e}$ versus $\varepsilon_{e}^{p}$ response for a proportional stress path test at high triaxiality $\eta$ in the triaxiality-insensitive regime. For the idealized cermets tested here we can take $\sigma_{0}\left(\varepsilon_{e}^{p}\right)$ to be the $\sigma_{e}$ versus $\varepsilon_{e}^{p}$ response for $\eta \geq 1$.

(iv) The viscoplastic parameters $\dot{\varepsilon}_{0}$ and $n$ are chosen to mimic a near rate-insensitive behaviour. Thus, following Peirce et al. [26] we take $n=20$ with $\dot{\varepsilon}_{0}$ chosen to be approximately equal to the strain rate employed in the experiments.

\subsection{Predictions of the proportional stress path responses}

The constitutive model is calibrated via uniaxial compression and $\eta=1$ proportional stress path tests. Model predictions for an intermediate triaxiality $\eta=0.75$ are presented in Fig. $8 \mathrm{~b}$ for both the mono-disperse and bi-disperse specimens. The material parameters used in the predictions (determined using the procedure outlined in section 4.1) are listed in Table 1 for

both specimen types, with $\sigma_{0}\left(\varepsilon_{e}^{p}\right)$ given by the $\sigma_{e}-\varepsilon_{e}^{p}$ responses for $\eta=1$ (see Figs. 3a and 7a for the mono and bi-disperse specimens, respectively). The model predictions are seen to be in good agreement with the measurements.

\begin{tabular}{ccc} 
& Mono-disperse & Bi-disperse \\
\hline$\sigma_{Y}(\mathrm{MPa})$ & 46.7 & 68.2 \\
$v$ & 0.33 & 0.33 \\
$E(\mathrm{GPa})$ & 33.0 & 46.6 \\
$\dot{\varepsilon}_{0}\left(\mathrm{~s}^{-1}\right)$ & $2 \times 10^{-5}$ & $2 \times 10^{-5}$ \\
$\mu$ & 0.7 & 0.9 \\
$n$ & 20 & 20
\end{tabular}

Table 1: Calibrated parameters of the phenomenological yield model. 


\section{Concluding remarks}

Axisymmetric compressive stress-strain responses have been measured for two idealized cermets, composed of mono or bi-dispersed steel spheres in a $\mathrm{Sn} / \mathrm{Pb}$ solder matrix. Triaxial tests were conducted under proportional and non-proportional stress paths. Non-proportional loading effects were found to be negligible and thus yield surfaces were extracted from proportional tests at a range of stress triaxialities. The responses of both types of cermets (stress-strain curves, dilation behaviour, yield surfaces) were qualitatively similar. However, the bi-disperse cermet exhibited a higher strength due to the larger volume fraction of steel spheres.

The initial yield strength of cermets is reasonably independent of the superimposed hydrostatic pressure, but the plastic hardening rate increases with stress triaxiality. However, beyond a critical value the compressive responses become insensitive to stress triaxiality. Two distinct deformation mechanisms give rise to this behaviour. At low triaxialities, the cermets behave as granular media and dilate under compressive loading. The increasing hardening rate with triaxiality is due to the work that must be done by the dilation mechanism against the superimposed hydrostatic pressure. By contrast, at sufficiently high triaxialities the deformation switches to a plastically incompressible mode, resulting in the observed insensitivity to stress triaxiality. These conclusions were further confirmed via X-ray tomography of specimens deformed under different triaxialities.

A viscoplastic phenomenological yield model that incorporates these deformation mechanisms was presented. The calibrated model was shown to be in good agreement with measurements. Currently there is no multi-axial data on high strength commercial cermets. The measurements presented here are suggestive of the deformation mechanisms that might 
be operative in such materials. We speculate that the proposed constitutive model can be calibrated by appropriate measurements to represent the response of commercial cermets.

\section{Acknowledgements}

The authors are grateful to the Office of Naval Research (ONR) for their financial support through grant number N000141210637 on Nickel bonded TiC composites for dynamic blast mitigation applications (program manager Dr. David Shifler). The authors would also like to acknowledge the discussions with Prof. Andreas Mortensen and Dr. Gabriella Tarantino which helped guide the study.

\section{References}

[1] ASTM Committee C- 21, "Report of Task Group B on Cermets," American Society for Testing and Materials, Philadelphia, PA, 1955, Phili, 1955.

[2] J.R. Tinklepaugh, W.B. Crandall, Cermets, Reinhold Publishing Company, New York, 1960.

[3] Z.Z. Fang, Correlation of transverse rupture strength of WC-Co with hardness, Int. J. Refract. Met. Hard Mater. 23 (2005) 119-127. doi:10.1016/j.ijrmhm.2004.11.005.

[4] B.G. Compton, F.W. Zok, Impact resistance of TiC-based cermets, Int. J. Impact Eng. 62 (2013) 75-87. doi:10.1016/j.ijimpeng.2013.06.008.

[5] W.R. Blumenthal, G.T. Gray III, T.N. Claytor, Response of aluminium-infiltrated boron carbide cermets to shock wave loading, J. Mater. Sci. 29 (1994) 4567-4576.

[6] D. Han, J.J. Mecholsky, Fracture behavior of metal particulate-reinforced WC-Co composites, Mater. Sci. Eng. A. 144 (1991) 293-302. doi:10.1016/09215093(91)90236-G.

[7] L.J. Prakash, Application of fine grained tungsten carbide based cemented carbides, 
Int. J. Refract. Met. Hard Mater. 13 (1995) 257-264. doi:10.1016/02634368(95)92672-7.

[8] M. Kouzeli, A. Mortensen, Size dependent strengthening in particle reinforced aluminium, Acta Mater. 50 (2002) 39-51. doi:10.1016/S1359-6454(01)00327-5.

[9] D.J. Lloyd, Particle reinforced aluminium and magnesium matrix composites, Int. Mater. Rev. 39 (1994) 1-23. doi:10.1179/095066094790150982.

[10] S. Scudino, G. Liu, K.G. Prashanth, B. Bartusch, K.B. Surreddi, B.S. Murty, J. Eckert, Mechanical properties of Al-based metal matrix composites reinforced with Zr-based glassy particles produced by powder metallurgy, Acta Mater. 57 (2009) 2029-2039. doi:10.1016/j.actamat.2009.01.010.

[11] Z. Xia, F. Ellyin, G. Meijer, Mechanical behavior of Al2O3-particle-reinforced 6061 aluminum alloy under uniaxial and multiaxial cyclic loading, Compos. Sci. Technol. 57 (1997) 237-248.

[12] I.C. Getting, G. Chen, J.A. Brown, The strength and rheology of commercial tungsten carbide cermets used in high-pressure apparatus, Pure Appl. Geophys. 141 (1993) 545-577. doi:10.1007/BF00998345.

[13] K. Mandel, L. Krüger, R. Krause, M. Radajewski, The influence of stress state on the compressive strength of WC-Co with different Co contents, Int. J. Refract. Met. Hard Mater. 47 (2014) 124-130. doi:10.1016/j.ijrmhm.2014.07.011.

[14] V. Jayaram, A. Kronenberg, S.H. Kirby, D.J. Rowcliffe, R. Sinclair, Plastic deformation of WC-Co at high confining pressure, Scr. Metall. 20 (1986) 701-705. doi:10.1016/0036-9748(86)90495-3.

[15] A. Hauert, A. Rossoll, A. Mortensen, Fracture of high volume fraction ceramic particle reinforced aluminium under multiaxial stress, Acta Mater. 58 (2010) 3895-3907. doi:10.1016/j.actamat.2010.03.037. 
[16] E. Bele, V.S. Deshpande, The Compressive Response of Idealized Cermetlike Materials, J. Appl. Mech. 82 (2015). doi:10.1115/1.4029782.

[17] J. Desrues, G. Viggiani, Strain localization in sand: An overview of the experimental results obtained in Grenoble using stereophotogrammetry, Int. J. Numer. Anal. Methods Geomech. 28 (2004) 279-321. doi:10.1002/nag.338.

[18] C. San Marchi, F. Cao, M. Kouzeli, A. Mortensen, Quasistatic and dynamic compression of aluminum-oxide particle reinforced pure aluminum, Mater. Sci. Eng. A. 337 (2002) 202-211. doi:10.1016/S0921-5093(02)00035-7.

[19] M. Oda, H. Kazama, Microstructure of shear bands and its relation to the mechanisms of dilatancy and failure of dense granular soils, Géotechnique. 48 (1998) 465-481. doi:10.1680/geot.1998.48.4.465.

[20] R.M. German, Particle Packing Characteristics, Metal Powder Industries Federation, Princeton, 1989.

[21] V.S. Deshpande, N.A. Fleck, Isotropic constitutive models for metallic foams, J. Mech. Phys. Solids. 48 (2000) 1253-1283. doi:10.1016/S0022-5096(99)00082-4.

[22] A.N. Schofield, Mohr Coulomb error correction, Gr. Eng. 31 (1998) 30-32.

[23] D.C. Drucker, W. Prager, Soil mechanics and plastic analysis for limit design, Q. Appl. Math. 10 (1952) 157-165.

[24] S. Okamoto, Y. Nakazono, K. Otsuka, Y. Shimoitani, J. Takada, Mechanical properties of WC/Co cemented carbide with larger WC grain size, Mater. Charact. 55 (2005) 281-287. doi:10.1016/j.matchar.2005.06.001.

[25] J.R. Rice, On the Structure of Stress-Strain Relations for Time-Dependent Plastic Deformation in Metals, J. Appl. Mech. 37 (1970) 728. doi:10.1115/1.3408603.

[26] D. Peirce, C.F. Shih, A. Needleman, A tangent modulus method for rate dependent solids, Comput. Struct. 18 (1984) 875-887. doi:10.1016/0045-7949(84)90033-6. 


\section{Figure Captions}

Figure 1: Optical micrographs of the cross-section of the (a) mono-disperse and (b) bidisperse idealized cermets. Magnified views of small regions within the cross-section are included in each case with the Sn/Pb solder matrix, steel spheres and voids labelled.

Figure 2: (a) Sketch of the triaxial apparatus used to apply the axi-symmetric stress states. The inset shows a magnified view of the specimen with the axial and radial LVDTs used to measure strains. (b) Sketch of the axi-symmetric stress state.

Figure 3: (a) The effective stress $\sigma_{e}$ versus effective strain $\varepsilon_{e}$ response for proportional loading of the mono-disperse specimens at selected values of the triaxiality $\eta$; (b) The volumetric strain $\varepsilon_{m}$ versus $\varepsilon_{e}$ responses corresponding to the curves in (a).

Figure 4: $X$-ray tomographic images of a longitudinal mid-section scan of mono-disperse specimens subjected to (a) uniaxial compression with $\eta=1 / 3$ and (b) high triaxiality proportional loading with $\eta=1.0$. In each case, sections prior to deformation $\left(\varepsilon_{e}=0\right)$, and after straining to $\varepsilon_{e}=5 \%$ are included. Magnified views of selected regions are also included in each case, with arrows marking newly formed voids and collapsing voids included in (a) and (b), respectively. 
Figure 5: The non-proportional (a) $\eta(1 / 3 \rightarrow 1)$ and (b) $\eta(1 \rightarrow 1 / 3)$ responses of the mono-disperse specimens plotted in terms of the measured effective stress $\sigma_{e}$ versus axial strain $\varepsilon_{a}$. In each case the proportional $\eta=1 / 3$ and $\eta=1$ curves are included for comparison purposes: any differences between measurements with the same $\eta$ value lie within the scatter due to small differences between test specimens. The inset shows the stress trajectory corresponding to points (i)-(iii) in $\sigma_{m}-\sigma_{e}$ space.

Figure 6: (a) The measured yield surfaces of the mono-disperse specimens. The initial yield surface and the evolution of the surface for 3 additional values of the effective plastic strain $\varepsilon_{e}^{p}$ are included. (b) Comparison between the initial and $\varepsilon_{e}^{p}=1 \%$ yield surfaces of the mono and bi-disperse specimens. The plastic strain rate vectors are included for selected loading points with the $\dot{\varepsilon}_{e}$ and $\dot{\varepsilon}_{m}$ axes co-incident with the $\sigma_{e}$ and $\sigma_{m}$ axes respectively in both (a) and (b).

Figure 7: (a) The effective stress $\sigma_{e}$ versus effective strain $\varepsilon_{e}$ response for proportional loading of the bi-disperse specimens at selected values of the triaxiality $\eta$. (b) The volumetric strain $\varepsilon_{m}$ versus $\varepsilon_{e}$ responses corresponding to the curves in (a).

Figure 8: (a) Sketch of the yield surface of the idealized cermet as the inner envelope of the set of lines corresponding to critical stressing of the granular and incompressible deformation mechanisms. The evolution of the yield surface with increasing effective plastic strain $\varepsilon_{e}^{p}$ is also indicated. (b) Comparison between experimental measurements and predictions of the constitutive model for the responses of mono and bi-disperse specimens at $\eta=0.75$. 


\section{Figure 1}

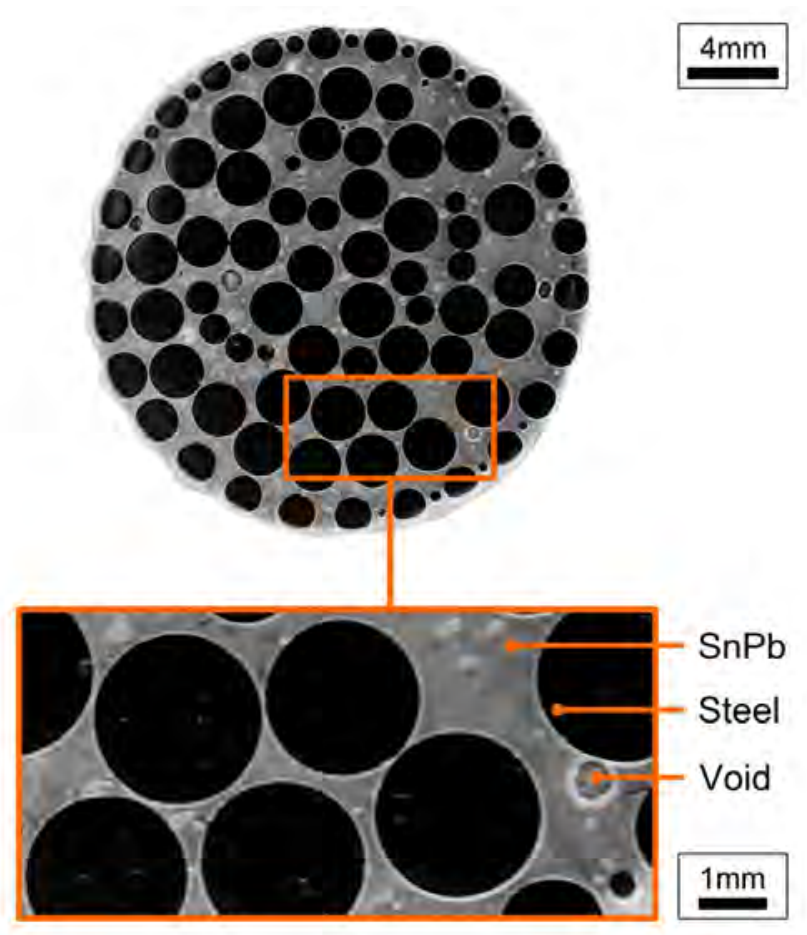

(a)

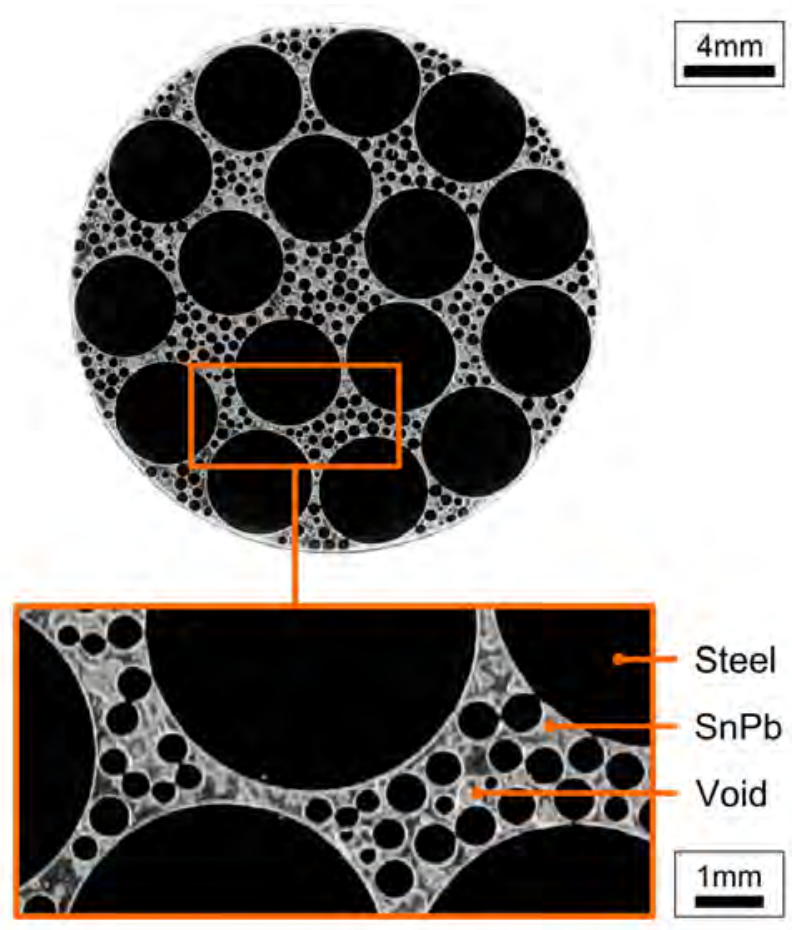

(b) 
Figure 3

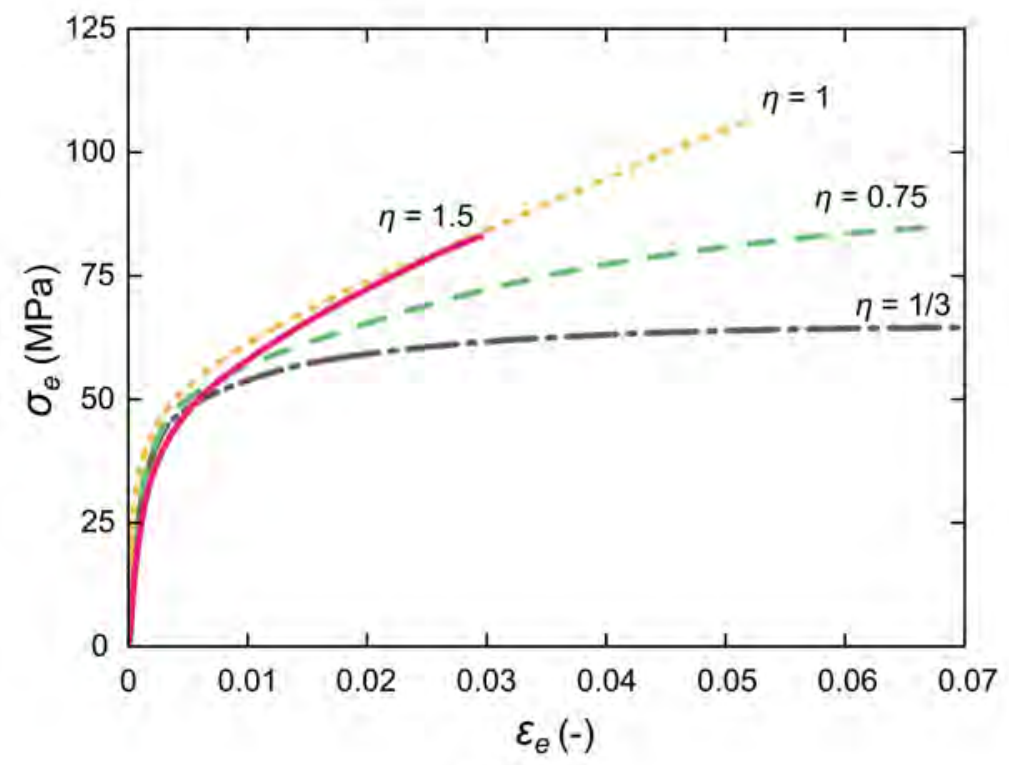

(a)

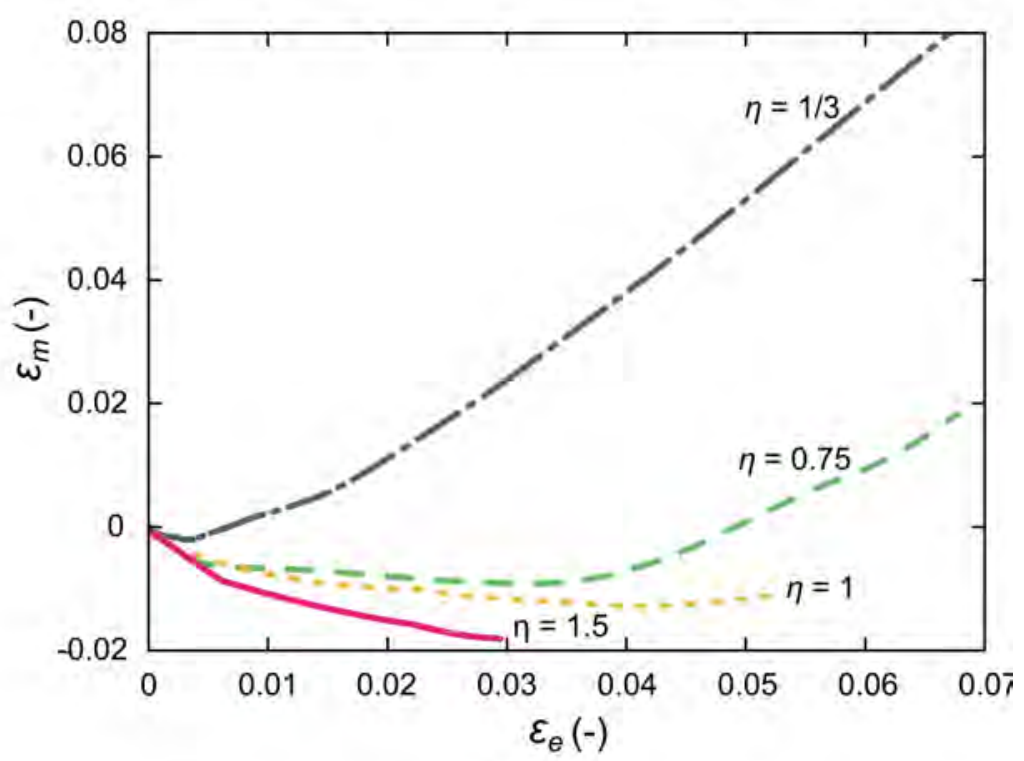

(b) 


\section{Figure 4}

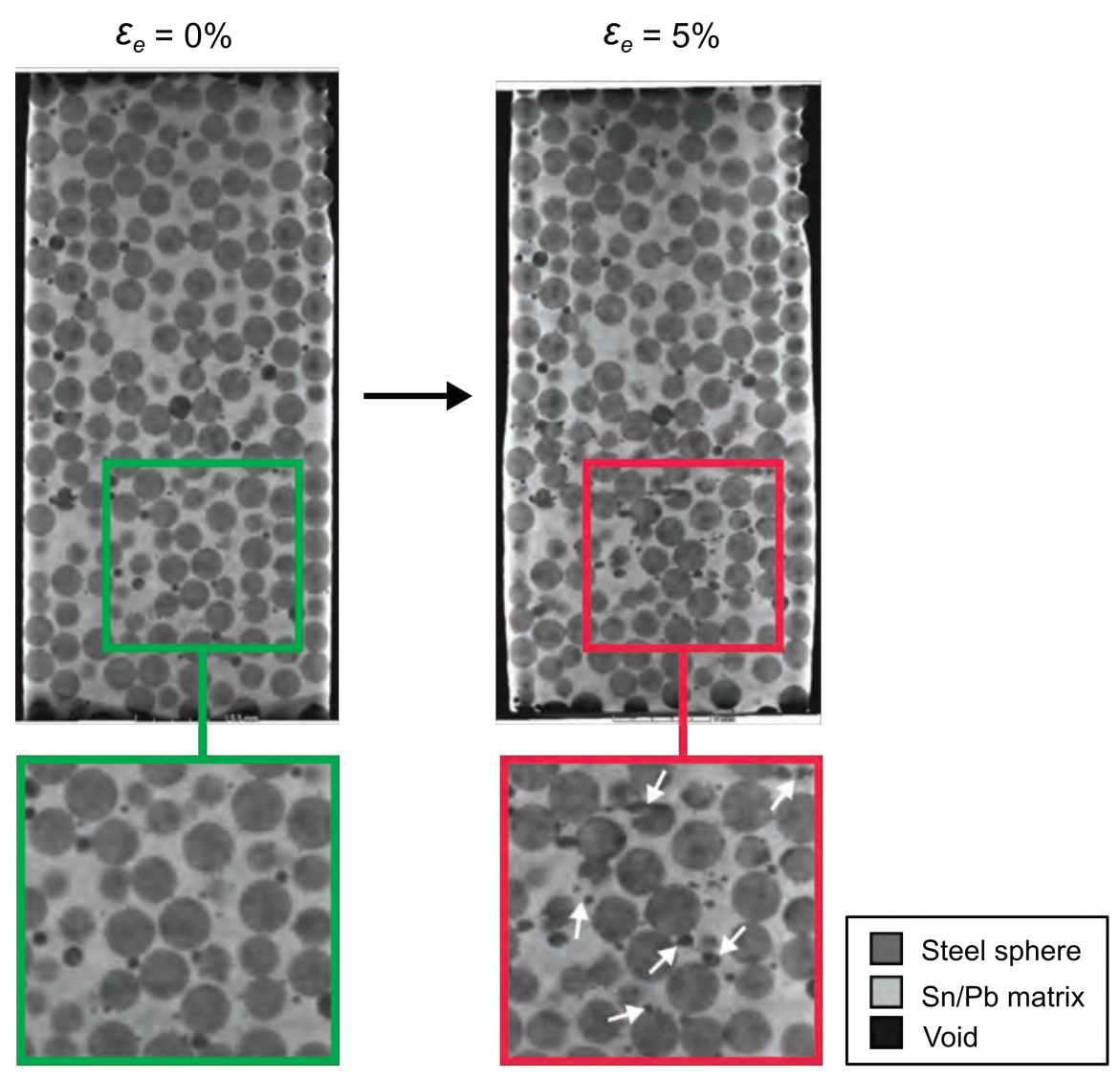

(a)

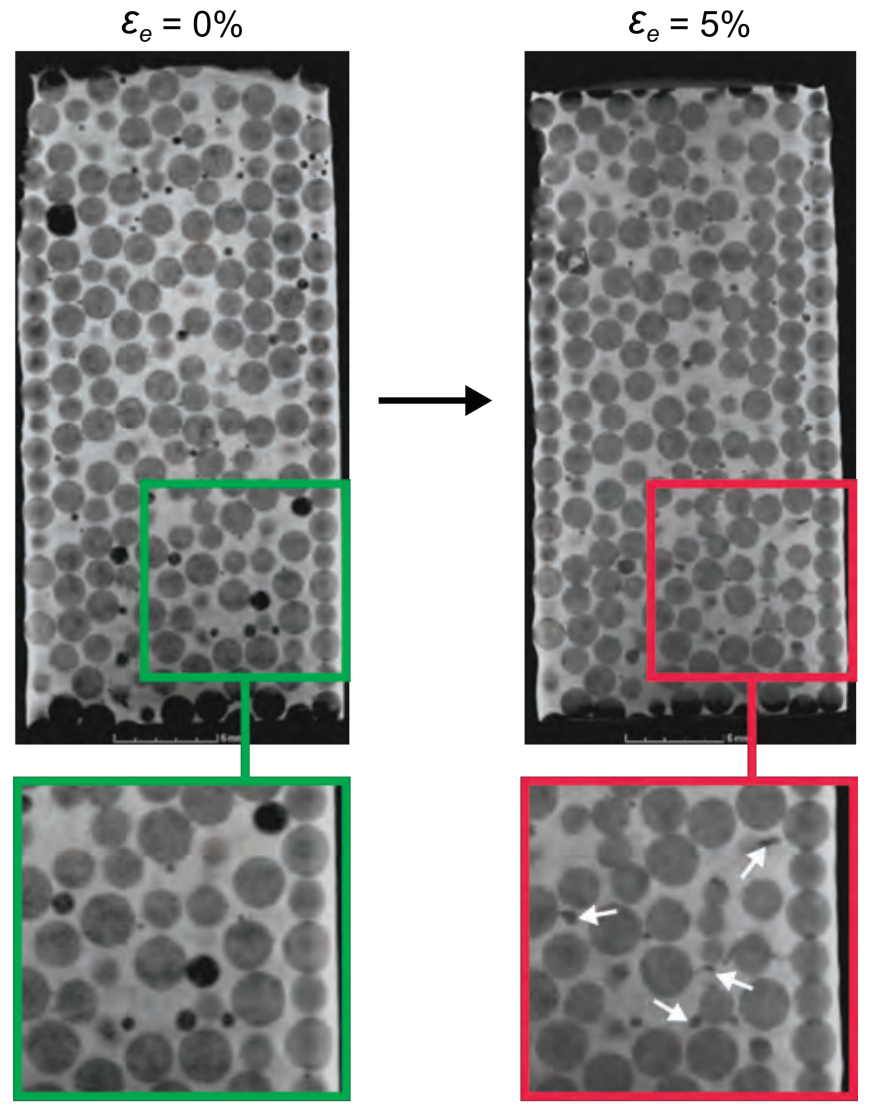

(b) 
Figure 5

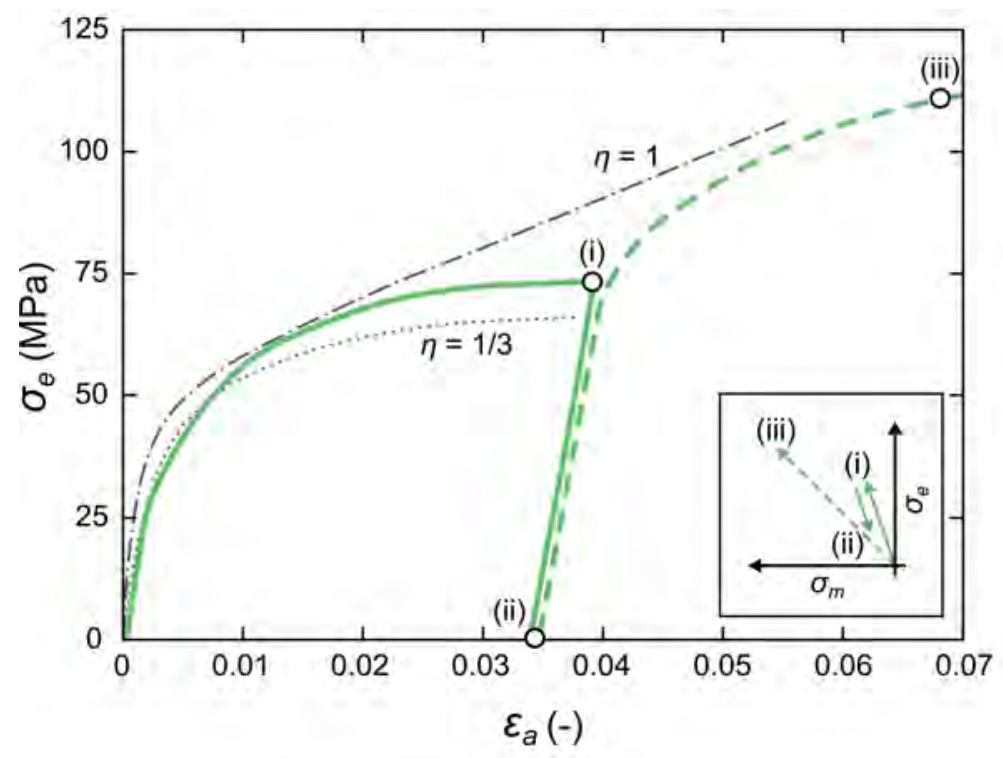

(a)

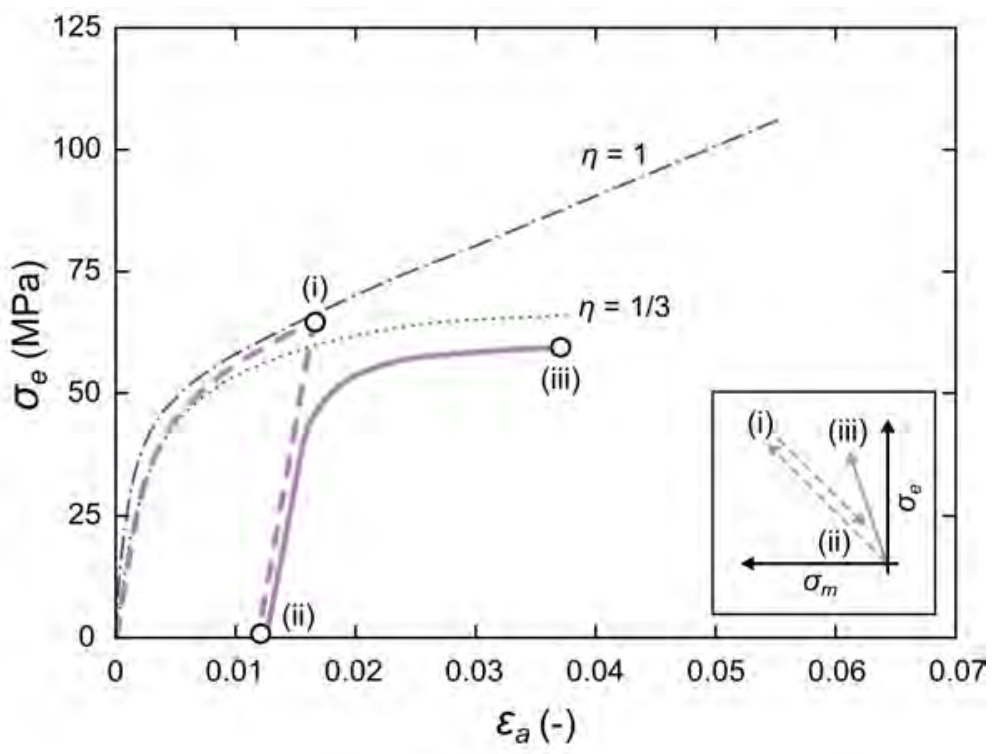

(b) 


\section{Figure 6}

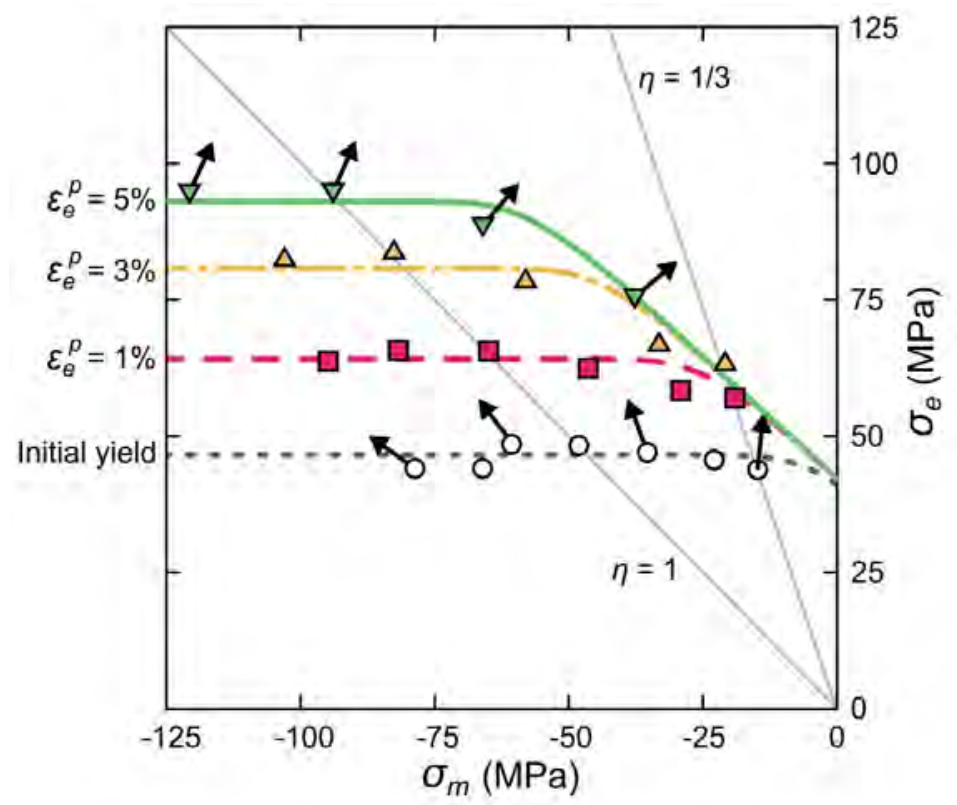

(a)

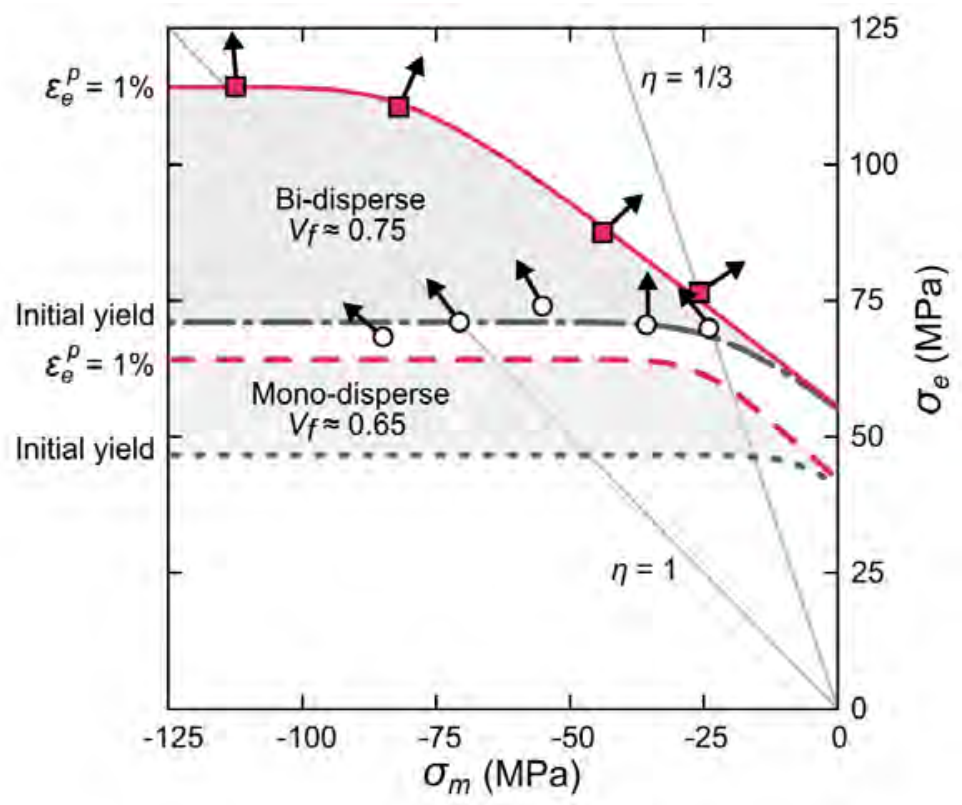

(b) 
Figure 7

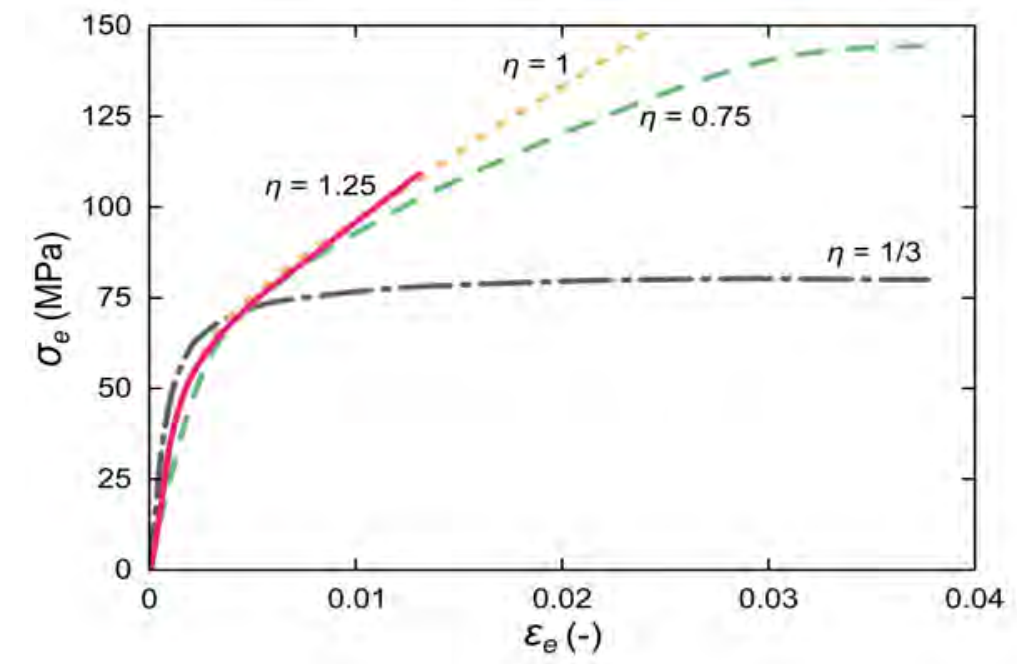

(a)

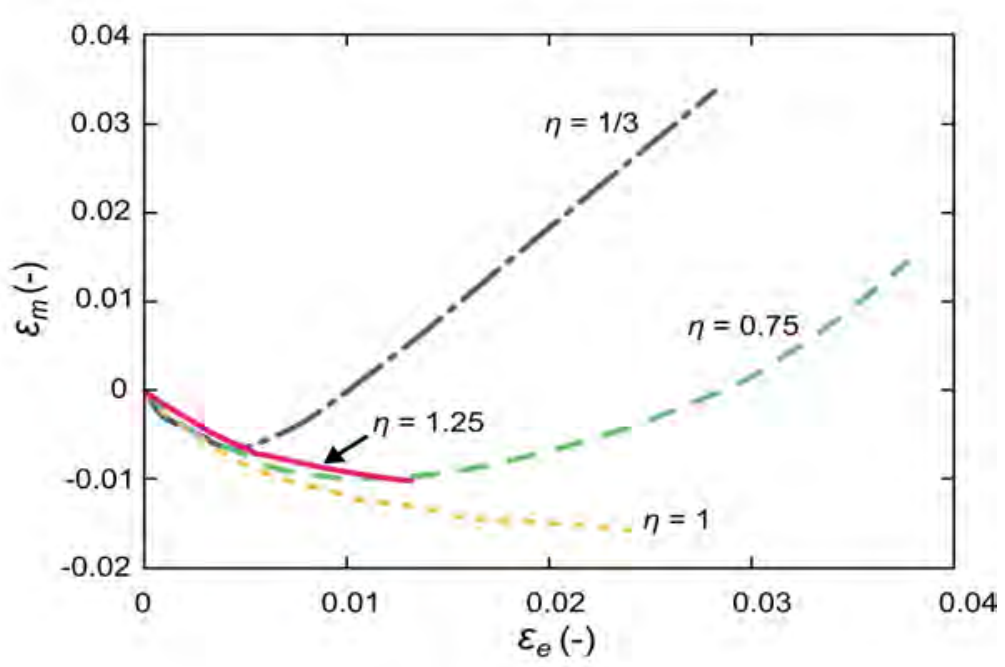

(b) 


\section{Figure 8}

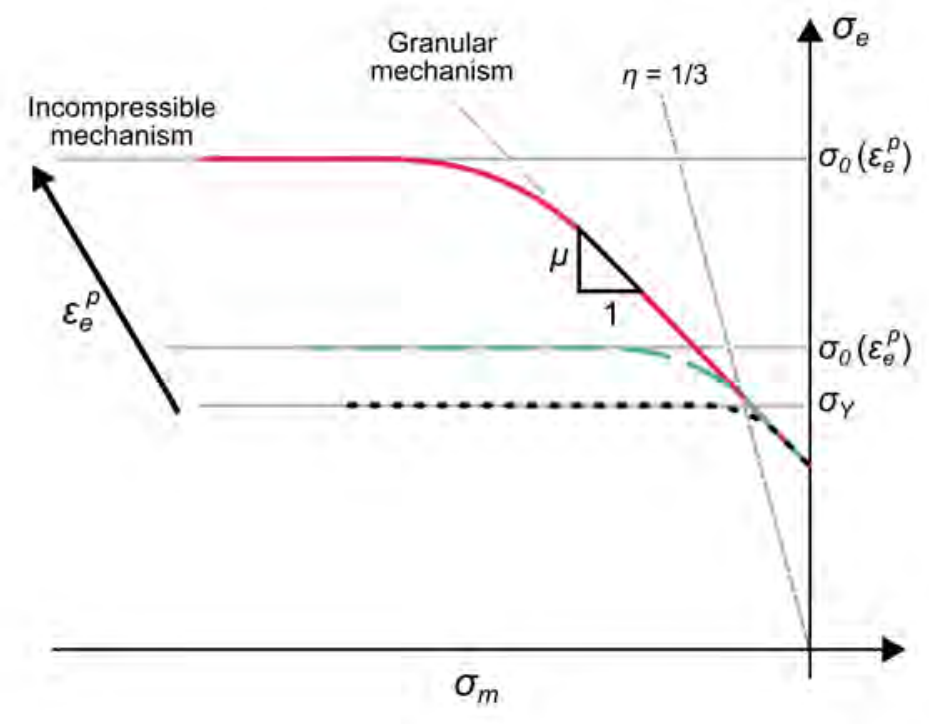

(a)

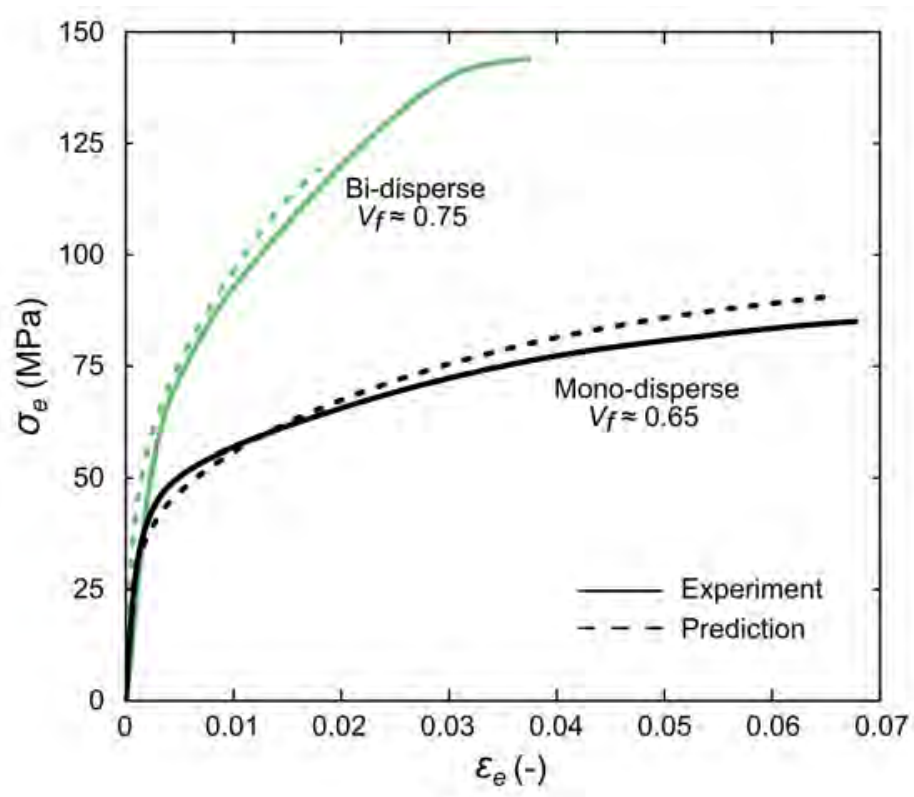

(b) 


\section{Graphical Abstract}
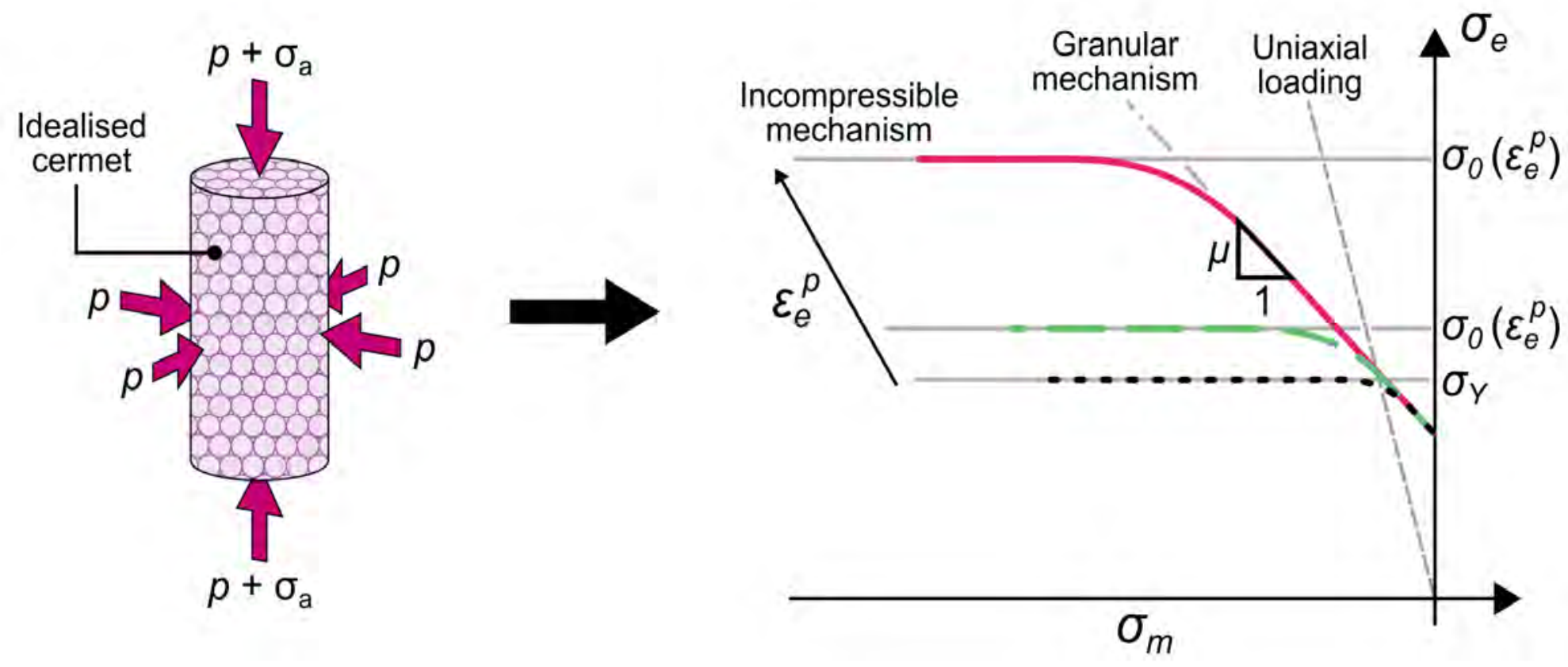